\title{
Anais da V Semana Acadêmica da Medicina - SAMED / UFSC - 2019
}

\author{
Josué Francisco da Silva Júnior ${ }^{1,2}$, André Vinícius Martinhago ${ }^{1,3}$, Alysson Nonato de Oliveira ${ }^{1,3}$, Gabriel Resun

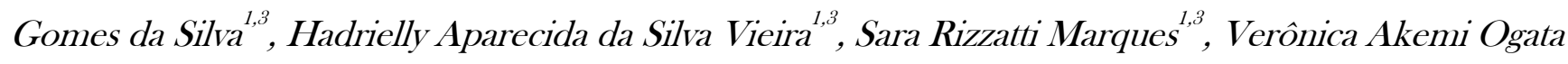 \\ Kawakatsu $^{1,3}$
}

1- Estudante do curso de graduação em Medicina, Centro de Ciências da Saúde, Universidade Federal de Santa Catarina (UFSC), Campus Reitor João David Ferreira Lima, Florianópolis, Brasil.

2- Diretor da Comissão de Trabalhos Científicos da $5^{\underline{a}}$ Semana Acadêmica da Medicina (SAMED-UFSC/2019)

3- Membro da Comissão de Trabalhos Científicos da $5^{\underline{a}}$ Semana Acadêmica da Medicina (SAMED-UFSC/2019)

Autor para correspondência: Josué Francisco da Silva Júnior. E-mail: josuefdsj@gmail.com Os autores declaram não terem conflitos de interesse em relação a esta publicação. Submetido em 02/11/19; aceito para publicação em 14/11/19

DOI: https://doi.org/10.32963/bcmufsc.v5i5.3823

$\mathrm{H}_{\mathrm{a}}$ á 5 anos, a Semana Acadêmica da Medicina (SAMED), evento marcado por 3 dias de palestras, workshops e oficinas, faz parte do calendário acadêmico oficial do curso de Medicina da Universidade Federal de Santa Catarina. Atualmente, em sua $5^{\circ}$ edição, além de toda a programação, contou também com o já tradicional momento científico - espaço no qual os alunos em conjunto com professores, residentes e médicos podem divulgar seus trabalhos científicos produzidos nos diversos espaços do curso (iniciações científicas, trabalhos de conclusão de curso, estágios e ligas acadêmicas).

O momento científico foi composto de três etapas: seleção de resumos a serem apresentados, avaliação dos pôsteres produzidos e apresentação oral dos 3 primeiros colocados. Primeiramente, os 15 resumos submetidos foram avaliados por nossa banca avaliadora, a qual, a fim de contemplar integralmente o curso, foi composta por 3 docentes pertencentes ao corpo clínico e por 2 docentes pertencentes ao corpo de disciplinas básicas nesse ano. Dentre os 15 resumos submetidos, 9 foram selecionados para a segunda etapa: confecção e apresentação dos pôsteres para a banca avaliadora. Em tal fase houve a apresentação dos pôsteres por um autor de cada resumo, que foram avaliados por professores representante de cada eixo (básico e clínico), sendo computada uma nota a diversos atributos pré-definidos em edital como apresentação, originalidade, relevância científica, entre outros. A terceira etapa consistiu na apresentação em formato de slides dos três trabalhos mais bem colocados, somando-se a nota de submissão do resumo e a da apresentação do seu respectivo pôster no evento. Esse momento ocorreu na noite do último dia do evento e que foi encerrada com a entrega da premiação para os respectivos autores dos trabalhos.

O momento científico contou com o apoio de toda a diretoria da V Semana Acadêmica de Medicina (SAMED), dos membros do Comitê de Trabalhos Científicos (CTC-SAMED), do centro Acadêmico Livre da Medicina da UFSC (CALIMED) e de nossos patrocinadores. Além disso, tal espaço também não existiria sem a participação ativa de nossos docentes que compuseram a banca avaliadora, ficando nossa eterna gratidão aos seguintes professores: Fabrício de Souza Neves, Jane da Silva, Juliana Dal-Ri Lindenau, Mariana Graciela Terenzi e Rosemeri Maurici da Silva.

Por fim, ao longo desta edição do Boletim da Medicina, você poderá contemplar os trabalhos de alguns de nossos participantes do momento científico. Esperamos que a produção de nossos colegas possa contribuir ainda mais para 0 seu aprendizado e ampliação do seu conhecimento.

"Aprender é a única coisa que a mente nunca se cansa, nunca tem medo e nunca se arrepende." (Leonardo da Vinci) 


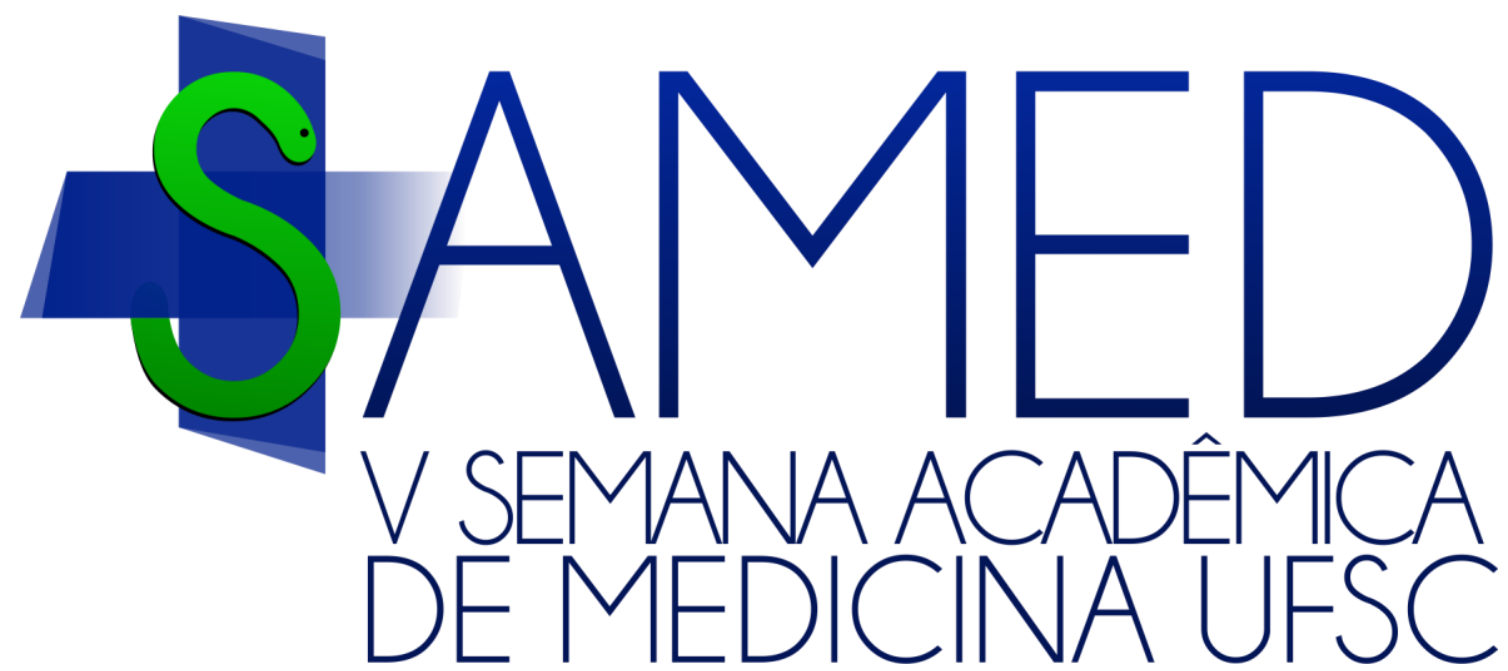

RESUMO EXPANDIDO 1 - V SAMED-UFSC/2019

\section{Doença pulmonar obstrutiva crônica e seu impacto na saúde pública}

Alexandre Holzbach $\mathrm{Jr}^{1}$, Ana Beatriz Goedert ${ }^{1}$, André Cardoso José1, Michele Mistura1, Rebeca Bernardi Heyse $^{1}$, Samuel dos Santos Bueno1, Valquíria Feier Goulart $^{1}$

1.Estudante do curso de graduação em Medicina, Centro de Ciências da Saúde, Universidade Federal de Santa Catarina (UFSC), Campus Reitor João David Ferreira Lima, Florianópolis, Brasil.

Objetivo. Este trabalho tem por objetivo realizar uma breve revisão não sistemática sobre a Doença Pulmonar Obstrutiva Crônica (DPOC) e identificar seu impacto na saúde pública do Brasil nos últimos anos, analisando-se quais as políticas públicas mais efetivas e os principais desafios encontrados no combate à doença. Metodologia: Analisaram-se artigos de bases de dados científicas como Scielo e PubMed, além de dados da Organização Mundial da Saúde (OMS), Instituto Brasileiro de Geografia e Estatística (IBGE), Ministério da Saúde (MS), Associação Brasileira de Saúde Coletiva (ABRASCO) e do Instituto Nacional de Combate ao Câncer (INCA). Resultados. A DPOC é uma enfermidade que compreende o enfisema pulmonar e a bronquite crônica, estando fortemente ligada ao tabagismo. É a $3 a$ maior causa de mortes no mundo, sendo que, destas, cerca de 85 a $90 \%$ são causadas pelo tabagismo, cujas toxinas causam alterações morfofuncionais no trato respiratório, levando à produção excessiva de muco, inflamação crônica das vias aéreas com consequente obstrução do fluxo aéreo e destruição do tecido pulmonar. Com isso, o paciente apresenta dificuldades nos movimentos respiratórios, levando a um quadro de acidose respiratória e sintomas como fadiga, dispneia e tosse prolongada com expectoração e sibilos. No Brasil, a DPOC atinge $12 \%$ da população acima de 40 anos, gerando 7 milhões de óbitos por ano e um gasto de $\mathrm{R} \$ 16$ bilhões pelos setores público e privado. Já que não há cura para a DPOC, tendo os tratamentos apenas a função de reduzir os sintomas e retardar a progressão da doença, a forma mais efetiva de combatê-la é a prevenção, a qual se aplica em três principais frentes: controle do tabagismo, investimento no diagnóstico precoce e promoção da saúde. Em relação ao controle do tabagismo, o Brasil, com o aumento das políticas de combate ao fumo nos últimos anos, obteve uma redução expressiva das mortes por DPOC no país, passando de 64,5/100.000 habitantes, em 1990, para 44,5 , em 2015 , com redução de $31 \%$ (32,6\% para o sexo masculino e $29,2 \%$ para o feminino). Esses dados coincidem com a queda de $57 \%$ e $56 \%$ na prevalência do tabagismo no mesmo período para homens e mulheres, respectivamente. Tais conquistas estão atrelada à adoção de diversas medidas preconizadas pela OMS, em especial 0 aumento dos impostos sobre o cigarro (hoje equivalentes a $80,42 \%$ do preço do produto), proibição do fumo em locais públicos, proibição da venda a menores de idade (pois $70 \%$ a $80 \%$ dos fumantes iniciam o hábito ainda na adolescência), proibição de campanhas publicitárias de cigarro e inclusão de advertências nas embalagens do produto alertando sobre os perigos inerentes ao hábito. Dentre as medidas, 0 aumento de impostos sobre o cigarro tem sido, certamente, a principal estratégia, pois, isoladamente, foi responsável por $50 \%$ da queda no tabagismo entre os brasileiros. Todas essas ações, por terem surtido um grande efeito em pouco tempo, tornaram o Brasil referência mundial no combate ao tabagismo, dando ao país papel de liderança na Convenção-Quadro para 0 Controle do Tabaco, tratado proposto em 1999 pelos países membros da Organização das Nações Unidas, com objetivo de deter a epidemia do tabagismo em todo o mundo. Mesmo assim, constatou-se que o tabagismo persiste como um desafio, pois ainda está presente em $9,3 \%$ da população brasileira. Além disso, embora tenha havido conquistas para a redução da DPOC em termos absolutos, em relação a outras doenças, a doença pulmonar obstrutiva crônica tem se tornado cada vez mais relevante no Brasil, visto que em 1990 era a 8 a principal causa de morte no país, passando para a $5^{\mathrm{a}}$ posição em 2017. Quanto à promoção do diagnóstico precoce, configura-se como um dos principais desafios no combate à doença, já que as taxas de subdiagnóstico da DPOC no Brasil chegam a $88 \%$, em grande parte resultado do despreparo de muitos médicos, os quais, não raro, consideram erroneamente a tosse do tabagista como algo normal, confundem os sintomas da DPOC com outras enfermidades e, em muitos casos, possuem dificuldade de 
interpretação e desconhecimento a respeito do principal método de diagnóstico da doença, a espirometria. Já em relação à última medida preventiva, a promoção da saúde, estima-se que $55 \%$ da população brasileira desconheça a doença, ressaltando a importância de conscientização. Um enfoque tem sido dado à trabalhos educativos nas escolas e entre os adolescentes, pois, como supracitado, a maioria dos indivíduos inicia o hábito tabagista antes da vida adulta e pesquisas indicam que $18,4 \%$ dos alunos do ensino fundamental no Brasil já tenham experimentado 0 uso de tabaco. Conclusão. Nas últimas décadas, Brasil conseguiu reduzir significativamente o número de mortes por DPOC, devido, principalmente, ao êxito de suas medidas de combate ao tabagismo, tanto preventivas, como educativas e regulatórias. Mesmo assim, a DPOC permanece como um grave problema de saúde pública no país, havendo ainda muitos desafios para combatê-la. Assim, maiores esforços visando melhorias no diagnóstico precoce, redução mais significativa do tabagismo e conscientização da população e da comunidade médica tornam-se necessários para a mitigação da doença.

\section{RESUMO EXPANDIDO 2 - V SAMED-UFSC/2019}

\section{Como evidenciar os efeitos da acupuntura no tratamento da dor neuropática? Relato da utilização da ferramenta de "Symptom Drawing"}

\section{Kamila Blaka1 ${ }^{1}$ Ari Ojeda Ocampo Moré2,3, Rina Dambi Yang Lee 2,3, Aluísio Uda 2,3, Li Shih Min 2,3,4}

1. Estudante do curso de graduação em Medicina, Centro de Ciências da Saúde, Universidade Federal de Santa Catarina (UFSC), Campus Reitor João David Ferreira Lima, Florianópolis, Brasil.

2. Médico do Serviço de Medicina Integrativa e Acupuntura do Hospital Universitário Prof. Polydoro Ernani de São Thiago (HUUFSC/EBSERH)

3. Preceptor da Residência Médica em Acupuntura do Hospital Universitário Prof. Polydoro Ernani de São Thiago (HUUFSC/EBSERH)

4. Professor do Departamento de Clínica Médica, Centro de Ciências da Saúde, Universidade Federal de Santa Catarina (UFSC), Campus Reitor João David Ferreira Lima, Florianópolis, Brasil.

Introdução. A escala visual analógica (EVA) é amplamente utilizada para quantificação de dor, porém esta limita-se apenas a aferir a intensidade da dor. Uma ferramenta complementar à EVA é o "Symptom Drawing" (SD). Este permite que o paciente, através desenhos coloridos em diagramas corporais, localize precisamente a extensão das áreas de dor e suas características dolorosas a longo da área acometida, de forma a complementar o quadro álgico com informações visuais. Considerando que trabalhos prévios demonstram que a acupuntura promove pouca modificação na EVA em casos de dor neuropática, o SD é uma ferramenta ainda pouco explorada nos estudos clínicos e que tem 0 potencial de mensurar de forma mais precisa os benefícios do tratamento com acupuntura. Objetivo. Descrever caso clínico de paciente com dor neuropática tratado com acupuntura e acompanhado através das ferramentas de EVA e SD. Metodologia. Um paciente de 38 anos apresentando dor neuropática miofascial pós-hernioplastia inguinal bilateral, com intervenções com evolução de 3 anos e refratário ao manejo farmacológico inicial com opioides, antidepressivos e anticonvulsivantes foi tratado no Ambulatório de Acupuntura do HU-UFSC. A hernioplastia bilateral foi feita por via de videolaparoscopia e uso de tela de polipropileno, em julho de 2015. Em junho de 2016 houve nova intervenção cirúrgica por via convencional para retirada dos grampos que foram utilizados para a fixação da tela. Bloqueio anestésico regional do nervo íleo inguinal e bloqueio no plano transverso do abdome guiados por Ultrassom, com melhora apenas por poucos dias. Realizou-se 4 ciclos de sessões de acupuntura (8 a 10 sessões por ciclo, associando-se técnicas de acupuntura manual e eletroacupuntura) com intervalo aproximado de 1 mês entre os ciclos no período de Janeiro de 2017 a Julho de 2018. No acompanhamento do caso utilizou-se a EVA e o aplicativo de SD "Catch my Pain $®$ ". Resultados. Inicialmente o paciente apresentava dor EVA 8 e esta estendia-se da região abdominal desde a altura da cicatriz umbilical, regiões inguinais até testículos (sintomas mais intensos à direita) com área de alodinia em quadrante inferior direito do abdome até a região inguinal. Ainda, 0 paciente complementou a avaliação com descritores como sensação de "queimação" e posição de alívio das dores em decúbito lateral e dorsal. Através da utilização de pontos de acupuntura segmentares e extra segmentares (na região lombar e membros), desativação dos pontos-gatilho em músculos quadrado lombar e iliopsoas, o paciente evoluiu com melhora progressiva da dor neuropática. No final do quarto ciclo de tratamento o paciente referia dor EVA 5, contudo foi expressiva redução da área total de dor e regiões de alodinia registradas através do SD, com analgesia importante das dores. Foi registrada na ferramenta de SD uma redução de aproximadamente $60 \%$ do território doloroso ao fim do tratamento, bem como a diminuição dos episódios de intensificação da dor quando comparado ao início do tratamento. Conclusão. A ferramenta de SD demonstrou ser útil no acompanhamento da melhora das queixas álgicas alcançadas pelo tratamento com acupuntura. Esta ferramenta auxiliou o paciente a descrever a sensação dolorosa com maior precisão do que a EVA. A inclusão do SD em estudos clínicos tem o potencial de melhor evidenciar os efeitos da acupuntura em pacientes com dor neuropática.

\section{RESUMO SIMPLES 1 - V SAMED-UFSC/2019}

\section{Osteogênese imperfeita: causas, diagnóstico e tratamento a partir de uma revisão da literatura}

Leonardo Busanello Mata Alves', Leonardo Busch Justino', Eduarda Gregório dos Santos Lima1, Gustavo Andreas Hochheim ${ }^{1}$, lago Koerich Silva ${ }^{1}$, Maria Luiza Toscan Camargo', Renata Guedes Garcia', Vinicius Cechella Borba ${ }^{1}$, Gabriel Adan Araujo Leite ${ }^{2}$

1. Estudante do curso de graduação em Medicina, Centro de Ciências da Saúde, Universidade Federal de Santa Catarina 
(UFSC), Campus Reitor João David Ferreira Lima, Florianópolis, Brasil.

2. Professor do Departamento de Biologia Celular, Embriologia e Genética, Centro de Ciências Biológicas, Universidade Federal de Santa Catarina (UFSC), Campus Reitor João David Ferreira Lima, Florianópolis, Brasil.

Objetivos. Realizar uma revisão da literatura sobre as causas, diagnósticos, tratamento e perspectivas da osteogênese imperfeita, considerada uma doença rara. Metodologia. Realizou-se uma pesquisa nas bases de dados Scielo, ScienceDirect e PubMed para obtenção de bibliografia, revisando detalhadamente o tema proposto. Resultados. Pesquisas mostraram que a osteogênese imperfeita (OI) afeta o tecido conjuntivo e a matriz extracelular, levando a um quadro de fragilidade óssea. Os trabalhos relatam as questões genéticas e hereditárias da Ol, condição rara que afeta 1:20 mil pessoas, com menos de 15 mil casos anuais no Brasil. Mutação em pelo menos um dos dois genes que codificam a proteína colágeno tipo I (COL1A1 e COL1A2) reduz a síntese de colágeno e/ou produz moléculas de colágeno disfuncionais. Os diagnósticos da doença são baseados no histórico individual do paciente, exames clínicos, constatações radiográficas, observação das fraturas e da fragilidade óssea. Já a classificação dos subtipos da doença depende do período em que as fraturas ocorreram e dograu de letalidade dos sintomas. A abordagem terapêutica da OI varia com a idade, gravidade da doença e estado funcional dos tecidos afetados. 0 tratamento inclui restrições sutis, como evitar atividades, e intensas intervenções ortopédicas, sendo a terapia com bifosfonatos intravenosos a terapêutica mais utilizada para tratar a fragilidade óssea em crianças - 0 efeito em adultos é pouco esclarecido. Conclusão. O estudo desse raro quadro clínico é de suma importância para o contínuo desenvolvimento de práticas terapêuticas que viabilizem o acréscimo da qualidade de vida dos indivíduos afetados por essa moléstia.

\section{RESUMO SIMPLES 2 - V SAMED-UFSC/2019}

\section{Avaliação da relação entre a qualidade de vida e a gravidade da urticária crônica espontânea e/ou induzida de pacientes atendidos no ambulatório de alergia do HU-UFSC}

Hadrielly Aparecida Da Silva Vieira1', Jordão Luiz Moratelli Junior ${ }^{1}$, Anne Rose Leopoldina Wiederkehr Baú2, Marcelo Martin Ferigato², Jane da Silva ${ }^{3}$

1. Estudante do curso de graduação em Medicina, Centro de Ciências da Saúde, Universidade Federal de Santa Catarina (UFSC), Campus Reitor João David Ferreira Lima, Florianópolis, Brasil.

2. Médico do Núcleo de Alergia do Hospital Universitário Prof.

Polydoro Ernani de São Thiago (HU-UFSC/EBSERH)

3. Professora do Departamento de Clínica Médica, Centro de Ciências da Saúde, Universidade Federal de Santa Catarina (UFSC), Campus Reitor João David Ferreira Lima, Florianópolis, Brasil.
Objetivos. Determinar a relação entre a gravidade da expressão dos sintomas e a qualidade de vida dos pacientes através de questionários validados. Metodologia. Análise retrospectiva de 2015 a 2019 de 61 pares de escores dos questionários Urticaria Activity Score 7 (UAS-7), que monitora a atividade da urticária, e o Dermatology Life Quality Index (DLQI), que avalia o impacto na qualidade de vida do paciente, de 23 pacientes. Realizaram-se estatísticas descritivas e análise estatística com teste qui-quadrado e 0 modelo linear de Poisson. Considerou-se $p<0,05$. Resultados. Dos 23 pacientes analisados $14(60,9 \%)$ eram adultos e 18 (78,3\%) eram do sexo feminino. O modelo linear de Poisson demonstrou que para cada aumento em um ponto no escore do UAS-7 observou-se um aumento de 6,4\% na pontuação do DLQI. Conclusão. foi observada uma relação estatisticamente significativa de impacto na percepção da qualidade de vida do paciente frente ao aumento da atividade da sua doença.

\section{RESUMO SIMPLES 3 - V SAMED-UFSC/2019}

Acesso e permanência de estudantes com deficiência em cursos da saúde: uma revisão integrativa

\section{Ruben Eduardo Krasniak ${ }^{1}$ Fabrício Augusto Menegon²}

1. Estudante do curso de graduação em Medicina, Centro de Ciências da Saúde, Universidade Federal de Santa Catarina (UFSC), Campus Reitor João David Ferreira Lima, Florianópolis, Brasil.

2. Professor do Departamento de Saúde Pública, Centro de Ciências da Saúde, Universidade Federal de Santa Catarina (UFSC), Campus Reitor João David Ferreira Lima, Florianópolis, Brasil.

Objetivo. Analisar o processo de acesso e permanência de estudantes com deficiência em cursos superiores da saúde. Método. Estudo de revisão integrativa da literatura a partir das bases eletrônicas BDENF, CINAHL, Lilacs, MEDLINE (através do PubMed e Web of Science), buscando artigos em português, inglês e espanhol, com filtro temporal de 10 anos. A busca foi efetuada em março de 2019. Utilizou-se como palavras chaves: "pessoas com deficiência", "estudantes de ciências da saúde" e descritores livres: "permanência estudantil", "inclusão", "política estudantil", "garantia a educação", "estratégias de enfrentamento", "estratégia de adaptação". Resultados. Na admissão, as instituições contam com normas técnicas excludentes, que focam na incapacidade e desconsideram o ensino com adaptações. Percebe-se a presença na comunidade acadêmica de um discurso negativo com relação a deficiência que considera esses alunos inaptos a proverem cuidado em saúde. Entre esses alunos, as deficiências mais prevalentes são de aprendizado e sensoriais. O suporte ao acadêmico enfatiza o ensino, com pouco auxílio em ambientes práticos de cuidado. A maioria desses estudantes não revelam a deficiência à instituição devido receio de serem discriminados. Não há relato de riscos à segurança do paciente assistido por estudantes com deficiência. Conclusão. Persistem muitas 
barreiras nesse processo de acesso e permanência dos estudantes com deficiência. Percebe-se uma exclusão sistematizada dessa população, com discriminação praticada por colegas e educadores. Outros estudos são necessários para melhor entender esse processo.

\section{RESUMO SIMPLES 4 - V SAMED-UFSC/2019}

\section{Cardiotoxicidade por intoxicação intencional com antidepressivo tricíclico: relato de caso}

\section{Maísa Maria Spagnol Trento1, Bruna Souza Marques, Adriana Mello Barotto², Jardel Jacinto²}

1. Estudante do curso de graduação em Medicina, Centro de Ciências da Saúde, Universidade Federal de Santa Catarina (UFSC), Campus Reitor João David Ferreira Lima, Florianópolis, Brasil.

2. Médico do Centro de Informação e Assistência Toxicológica da Secretaria de Estado da Saúde de Santa Catarina / Hospital Universitário Prof. Polydoro Ernani de São Thiago (HUUFSC/EBSERH)

Introdução. Os antidepressivos tricíclicos configuram-se como causa frequente de intoxicação intencional, situação clínica potencialmente grave. Objetivos. Relatar caso que destaca a cardiotoxicidade em intoxicação fatal por tricíclico. Descrição do caso. MGS, feminina, 67 anos, $60 \mathrm{~kg}$, trazida pelo SAMU em glasgow 3 por tentativa de suicídio, há 2 horas (h), com ingesta de $37,5 \mathrm{mg} / \mathrm{kg}$ de Nortriptilina (dose tóxica: $2,5 \mathrm{mg} / \mathrm{kg}$ ). Realizado lavagem gástrica, carvão ativado e intubação sem sequência rápida. Apresentava-se com pressão arterial (PA) $60 \times 40 \mathrm{mmHg}$ mesmo após hidratação e noradrenalina (nora) de $0,1 \mathrm{mcg} / \mathrm{kg} / \mathrm{min}$, realizado bolus $(1 \mathrm{meq} / \mathrm{kg}$ ) de bicarbonato de sódio ( $\mathrm{NaHCO}$ ) 8,4\% por QRS alargado em eletrocardiograma. Após $3 \mathrm{~h}$ da ingesta apresentou taquicardia ventricular (TV) sem pulso, procedido cardioversão elétrica e ajustado nora para $0,5 \mathrm{mcg} / \mathrm{kg} / \mathrm{min}$, mantendo PA 100x50 mmHg. Após $12 \mathrm{~h}$ da ingesta apresentou instabilidade hemodinâmica, TV não sustentada e convulsões. Foi iniciado fenitoína, com melhora das convulsões apenas após substituição por fenobarbital. Manteve TV não sustentada, apesar da infusão contínua de lidocaína e $\mathrm{NaHCO}(\mathrm{pH} 7,5)$. Com 24h da ingesta apresentou TV sustentada, torsades de pointes não cardiovertida. Após ajuste de $\mathrm{NaHCO} 3$, sulfato de magnésio e nora, normalizou ritmo e hemodinâmica, manteve QT longo. Com 36h após intoxicação, apresentou bloqueio atrioventricular total, choque refratário, parada cardiorrespiratória e óbito. Conclusão. A cardiotoxicidade dos tricíclicos é multifatorial: por inibição dos canais rápidos de sódio e da recaptação de catecolaminas, bloqueio alfa adrenérgico periférico e efeitos anticolinérgicos, sendo a bradicardia de mal prognóstico. Tais dados justificam as amplas manifestações das intoxicações e a importância de reconhecer as repercussões e potencial letalidade.

\section{RESUMO SIMPLES 5 - V SAMED-UFSC/2019}

\section{Regressão Espontânea de Metástases Após Nefrectomia Citorredutora}

\author{
Pietro Valentin Waltrick Bernardi1, Natália Nara Park \\ Andrade ${ }^{1}$, Carlos Fernando Bernardi2
}

1. Estudante do curso de graduação em Medicina, Centro de Ciências da Saúde, Universidade Federal de Santa Catarina (UFSC), Campus Reitor João David Ferreira Lima, Florianópolis, Brasil.

2. Médico oncologista, Clínica Bernardi, Concórdia, Santa Catarina, Brasil

Introdução. Desaparecimento completo de um tumor sem intervenção relevante é um fenômeno raro, no tumor primário ou em metástases. Apesar de mal compreendida, sua etiologia parece estar relacionada a fenômenos imunológicos. Não se sabe com que frequência regressões acontecem, mas aproximadamente 20 casos são registrados na literatura a cada ano. Destes, 0 carcinoma de células renais (RCC) é um dos tipos de tumor no qual isto é mais frequente. Descrição. Paciente realizara ultrassonografia (USG), revelando metástases hepáticas e massa renal esquerda. Posterior tomografia computadorizada (TC) confirmou lesões sugestivas de metástases hepáticas. TC tórax normal, cintilografia óssea normal, TC e ressonância magnética (RM) de crânio normais. Realizou nefrectómica esquerda citorredutora em 30/05/17. Paciente retornou 3 meses após a nefrectomia, com PET-CT normal. Solicitada RM, também normal. Retornou após 4 meses e após 8 meses, com TC de tórax e RM abdominal superior, ambas normais nas duas situações. Discussão. A relação da regressão com a cirurgia não pode ser ignorada - possivelmente a inflamação e 0 extravasamento de antígenos provocaram ativação da resposta imune adaptativa contra células tumorais. A literatura ainda é incapaz de fornecer conclusões concretas sobre as causas das regressões espontâneas, mas compreender as causas é crucial para o desenvolvimento de imunoterapias. Conclusão. É importante a publicação de relatos como este, com acompanhamento posterior, para definir probabilidades e características da regressão em cada tipo de tumor. A frequência relativamente alta da regressão espontânea de metástases no RCC pode ser um bom ponto de partida para estudos sobre os mecanismos imunológicos do fenômeno. 